\title{
Avaliação do estado nutricional em pacientes idosos oncológicos internados em um hospital de alta complexidade do Norte do Rio Grande do Sul
}

\author{
Assessment of nutritional status in oncological elderly patients admitted to a high \\ complexity hospital of the North of Rio Grande do Sul
}

Elisa Pelissaro ${ }^{1,2} \bowtie$, Cássia Cassol Damo ${ }^{1,2}$, Ana Luisa Sant'Anna Alves², Aline Calcing1, Daiana Argenta Kümpel ${ }^{2}$

${ }^{1}$ Hospital São Vicente de Paulo. Passo Fundo, RS.

2 Universidade de Passo Fundo (UPF). Passo Fundo, RS.

\section{RESUMO}

Objetivos: Avaliar o risco nutricional em pacientes idosos oncológicos hospitalizados.

Métodos: Estudo retrospectivo transversal realizado com pacientes idosos oncológicos internados no Hospital São Vicente de Paulo, em Passo Fundo, Rio Grande do Sul. Foram selecionados os pacientes com câncer que passaram pela avaliação antropométrica e subjetiva por meio da Mini Avaliação Nutricional Reduzida. Os dados foram coletados do formulário de investigação utilizado pelo Instituto Nacional de Câncer, incluindo avaliação de dados clínicos, tipo de câncer, motivo e tempo de internação.

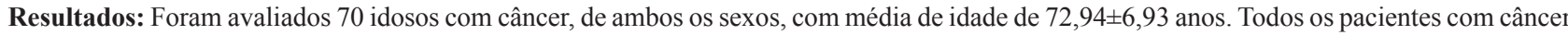
de pâncreas apresentaram perda de peso. Os pacientes com câncer de pulmão e de pâncreas foram os que apresentaram maior porcentagem de perda de peso grave (maior que $10 \%$ do peso habitual nos últimos seis meses), $57,1 \%$ e $40 \%$ respectivamente, enquanto a maioria dos casos de câncer de mama não tinham perda de peso. $\mathrm{O}$ índice de massa corporal e a circunferência da panturrilha diagnosticaram maior proporção de eutrofia (55,7\% e 65,7\%, respectivamente), diferentemente da Mini Avaliação Nutricional Reduzida, que identificou maiores percentuais de desnutrição ou risco nutricional $(84,4 \%)$.

Conclusões: A maioria dos idosos oncológicos hospitalizados encontravam-se eutróficos pelos indicadores antropométricos índice de massa corporal e circunferência da panturrilha, enquanto uma proporção maior desses pacientes foram classificados como desnutridos ou em risco nutricional pela Mini Avaliação Nutricional Reduzida, assim como pela porcentagem de perda de peso. Os pacientes com câncer de pulmão e pâncreas foram os que mais apresentaram perda de peso grave. Os presentes resultados confirmam a importância de usar diferentes parâmetros para avaliar o estado nutricional de idosos oncológicos.

DESCRITORES: idosos; neoplasia; estado nutricional.

\section{ABSTRACT}

Aims: To assess the nutritional risk in hospitalized oncological elderly patients.

Methods: Retrospective study conducted with oncological elderly patients hospitalized at the São Vicente de Paulo Hospital, in Passo Fundo, Rio Grande do Sul, Brazil. Patients with cancer who underwent anthropometric and subjective evaluation by the Mini Nutritional Assessment Reduced Forms were selected. Data were collected from the research form used by the National Cancer Institute, including clinical evaluation, type of cancer, reason for hospital admission and length of stay.

Results: A total of 70 elderly patients with cancer, of both genders, with a mean age of $72.94 \pm 6.93$ years, were evaluated. All patients with pancreatic cancer had weight loss. Patients with lung cancer and pancreatic cancer had the highest percentage of severe weight loss (greater than $10 \%$ of usual weight in the last six months), $57.1 \%$ and $40 \%$ respectively, while the majority of breast cancer cases had no weight loss. Body mass index and calf circumference diagnosed greater proportion of normal weight $(55.7 \%$ and $65.7 \%$, respectively), unlike the Mini Nutritional Assessment Reduced Forms, that identified higher rates of malnutrition or nutritional risk (84.4\%).

Conclusions: Most hospitalized oncological elderly were found to be eutrophic by the anthropometric indicators body mass index and calf circumference, while a higher proportion of these patients were classified as malnourished or at nutritional risk by the Mini Nutritional Assessment Reduced Forms, as well as by the percentage of weight loss. Patients with lung and pancreas cancer had the most severe weight loss. These results confirm the importance of using different parameters to assess the nutritional status of elderly with cancer.

KEY WORDS: elderly; neoplasia; nutritional status.

Recebido: fevereiro, 2016

Aceito: junho, 2016

Publicado: julho, 2016

Este artigo está licenciado sob forma de uma licença Creative Common 
Abreviaturas: HSVP, Hospital São Vicente de Paulo; MAN, Mini Avaliação Nutricional; MANR, Mini Avaliação Nutricional Reduzida; IMC, índice de massa corporal; CP, circunferência da panturrilha; INCA, Instituto Nacional do Câncer.

\section{INTRODUÇÃO}

De acordo com a Organização Mundial de Saúde, nos países desenvolvidos são considerados idosos os indivíduos com idade igual ou superior a 65 anos, enquanto nos países em desenvolvimento considerase o limite etário igual ou superior a 60 anos [1]. As transições na demografia mundial e o aumento da expectativa de vida têm levado ao envelhecimento populacional e ao aumento significativo da população idosa. Porém, cada vez mais as pessoas estão envelhecendo com enfermidades crônicas como o câncer, que passa a ocupar um espaço relevante no cenário epidemiológico mundial [2,3]. Estimase que 26 milhões de novos casos de câncer serão diagnosticados no mundo até 2030 , sendo que mais de $50 \%$ de todas as neoplasias e $70 \%$ da mortalidade ocorrerão em indivíduos acima dos 65 anos [2,4]. Segundo o Ministério da Saúde [5], nos últimos cinco anos, $65 \%$ dos óbitos por câncer no Brasil ocorreram em indivíduos na faixa etária de 60 anos ou mais.

O câncer é uma doença multifatorial, caracterizado pelo crescimento de células anormais, agressivas e incontroláveis, com tendência a invadir tecidos e órgãos [6]. Assim como outras doenças crônicas, o câncer é considerado prevenível, apresentando uma taxa de cura de $50 \%$. Entretanto, o prognóstico do paciente depende do diagnóstico precoce, planejamento e acompanhamento terapêutico adequado [7]. A localização da neoplasia, os demais órgãos acometidos, o diagnóstico precoce ou tardio, assim como as condições clínicas, imunológicas e nutricionais, são fatores que interferem com o estado geral do paciente, implicando em seu prognóstico e na efetividade do tratamento [8]. Os distúrbios metabólicos e o comprometimento do estado nutricional são frequentes em indivíduos com câncer, estando associados ao maior risco de morbimortalidade, maior susceptibilidade a infecções e tempo de hospitalização mais prolongado, piorando o prognóstico do paciente $[9,10]$.

Sob o ponto de vista nutricional, é necessário identificar os principais fatores de risco e fazer um diagnóstico oportuno de desnutrição ou de risco nutricional, por meio de avaliações subjetivas e/ou antropométricas. A Mini Avaliação Nutricional Reduzida (MANR) é um dos métodos subjetivos, sendo um instrumento de baixo custo e fácil aplicação, validado para avaliar o estado nutricional de idosos hospitalizados ou institucionalizados [11,12]. Entre os métodos de avaliação antropométrica, o Índice de Massa Corporal (IMC) é o parâmetro mais utilizado no rastreamento nutricional. O IMC identifica a alteração de peso em relação à altura, um importante dado para avaliação nutricional. No caso de pessoas com câncer, o percentual de perda de peso pode ter relação com mau prognóstico clínico [13]. Outro método antropométrico é a medida da circunferência da panturrilha (CP), também um importante marcador para a desnutrição, sendo associada à massa muscular. A CP é uma medida econômica e não invasiva, além de ser considerada como o melhor indicador clínico de sarcopenia e estar relacionada também à incapacidade funcional [14].

A avaliação, o acompanhamento e a assistência nutricional adequada ao paciente oncológico são fundamentais para detectar e prevenir carências nutricionais, bem como agravos no quadro clínico, propondo intervenções de forma precoce. As intervenções nutricionais podem melhorar a resposta ao tratamento, reduzir os riscos de complicações, diminuir a morbimortalidade e proporcionar melhor qualidade de vida ao paciente oncológico $[9,15]$.

Dessa forma, o presente estudo teve como objetivo utilizar diversos métodos de avaliação do risco nutricional em idosos oncológicos hospitalizados.

\section{MÉTODOS}

Foi realizado um estudo transversal retrospectivo, com utilização de dados secundários, no Hospital São Vicente de Paulo (HSVP), em Passo Fundo, Rio Grande do Sul, considerado um hospital completo de fins filantrópicos, que desenvolve atividades de atendimento médico-hospitalar sem finalidades lucrativas, atuando nas áreas de medicina de alta complexidade, internação hospitalar, serviços ambulatoriais, centro de oncologia, transplantes e engenharia biomédica.

Os dados foram obtidos por meio de um formulário elaborado pelo Instituto Nacional de Câncer (INCA) contendo informações clínicas, demográficas e do estado nutricional de pacientes oncológicos internados. Esse formulário foi utilizado para a construção do Inquérito Luso-Brasileiro de Nutrição Oncológica do Idoso [15] e teve como objetivo obter informações do estado nutricional de idosos oncológicos no período de setembro a novembro de 2014.

Foram avaliados todos os pacientes oncológicos internados no HSVP no período citado, com idade igual ou superior a 65 anos, de ambos os sexos, 
portadores de tumores malignos independentemente da localização ou estadiamento da doença, e com o estado nutricional avaliado nas primeiras 24 horas de internação. Pacientes em tratamento oncológico que não passaram pela avaliação nutricional não foram incluídos no estudo, uma vez que o formulário do INCA não havia sido preenchido para os mesmos.

No formulário de investigação do INCA constam as seguintes informações: identificação do paciente, tempo de internação, localizaçãodo câncer, motivo da internação (se clínico ou cirúrgico), dados antropométricos e avaliação nutricional subjetiva pela MANR.

A partir dos dados antropométricos peso em quilos (kg) e altura em metros (m) foi calculado o IMC $\left(\mathrm{kg} / \mathrm{m}^{2}\right)$. Para classificação do estado nutricional foram adotados os pontos de corte para idosos, proposto por Lipschitz [16]: IMC $<22 \mathrm{~kg} / \mathrm{m}^{2}$ - baixo peso; IMC entre 22 e $27 \mathrm{~kg} / \mathrm{m}^{2}$ - eutrofia; e IMC $>27 \mathrm{~kg} / \mathrm{m}^{2}$ - sobrepeso/ obesidade (peso excessivo).

Para verificar a porcentagem de perda de peso utilizou-se a fórmula (peso usual - peso atual) $\times 100 /$ peso usual, conforme Blackburn et al. [17]. Considerou-se ausência de perda de peso quando menos de $1 \%$, perda significativa de peso entre $1 \%$ e $10 \%$ e perda grave quando mais de $10 \%$ do peso nos últimos seis meses. O peso usual foi estimado pela informação do próprio paciente, que se encontrava anotada na ficha.

A classificação do estado nutricional segundo a $\mathrm{CP}$ seguiu os parâmetros propostos por Chumlea et al. [18] para homens e para mulheres, sendo $\mathrm{CP} \geq 31 \mathrm{~cm}-$ eutrofia; e $\mathrm{CP}<31 \mathrm{~cm}$ - marcador de desnutrição.

A MANR é constituída por seis itens, abrangendo aspectos alimentares (perda de apetite), antropométricos (IMC e perda de peso), e aspectos relacionados à condição de saúde (mobilidade, problemas neuropsicológicos e estresse psicológico). $\mathrm{O}$ estado nutricional é classificado de acordo com a soma obtida em cada item, em três escores: desnutrição (0 a 7 pontos); risco de desnutrição (8 a 11 pontos) e estado nutricional normal (12 a 14 pontos) [19].

O idoso oncológico foi considerado em risco nutricional quando pelo menos um dos parâmetros antropométricos esteve abaixo da normalidade, ou pela pontuação da MANR com diagnóstico de risco de desnutrição ou desnutrição instalada.

Os dados coletados foram tabulados em planilhas do Microsoft Office Excel 2010 e analisados pelo IBM SPSS versão 18.0. Para as análises de associação foi aplicado o teste qui quadrado, considerando o nível de significância de $95 \%$.

O estudo foi aprovado pelo Comitê de Ética em Pesquisa da Universidade de Passo Fundo sob parecer de número 1.162.505. Os pesquisadores comprometeram-se a assegurar a privacidade dos sujeitos da pesquisa e utilizar as informações somente de forma anônima e exclusivamente para execução do estudo.

\section{RESULTADOS}

Foram avaliados 70 pacientes idosos oncológicos com média de idade $72,94 \pm 6,93$ anos (mínima 65 anos e máxima 91 anos). A localização mais frequente do câncer foi o intestino (70\%). Características dos pacientes estudados estão descritas na Tabela 1.

Tabela 1. Perfil demográfico e clínico de idosos oncológicos internados no Hospital São Vicente de Paulo em Passo Fundo, RS, $2014(n=70)$.

\begin{tabular}{lccc}
\hline \multicolumn{1}{c}{ Variáveis } & Categoria & $\mathbf{n}$ & $\mathbf{( \% )}$ \\
\hline Sexo & Feminino & 30 & $(42,9)$ \\
& Masculino & 40 & $(57,1)$ \\
Faixa etária & $65-69$ anos & 28 & $(40)$ \\
& $70-79$ anos & 31 & $(44,3)$ \\
& $\geq 80$ anos & 11 & $(15,8)$ \\
Motivo da internação & Cirúrgico & 31 & $(44,3)$ \\
& Clínico & 39 & $(57,7)$ \\
Tempo de internação* & $1-10$ dias & 67 & $(95,7)$ \\
& $>30$ dias & 3 & $(4,3)$ \\
Localização do câncer & Intestino & 18 & $(25,7)$ \\
& Próstata & 11 & $(15,7)$ \\
& Pulmão & 7 & $(10)$ \\
& Pâncreas & 7 & $(10)$ \\
& Mama & 6 & $(8,6)$ \\
& Outros & 21 & $(30)$ \\
\hline
\end{tabular}

* Nenhum paciente esteve internado entre 11 e 30 dias.

O valor médio do IMC com o respectivo desvio padrão foi de $25,51 \pm 4,85 \mathrm{~kg} / \mathrm{m}^{2}$. Segundo esse método antropométrico, 12 pacientes $(17,1 \%)$ apresentavam diagnóstico de desnutrição. Considerando-se a classificação nutricional pela CP, 24 pacientes $(34,3 \%)$ foram classificados com desnutrição. Não houve diferença significativa entre os sexos em relação ao IMC ou à CP (Tabela 2).

Obteve-se a informação sobre perda de peso nos últimos seis meses de 63 pacientes, sendo que sete pacientes não haviam informado seu peso usual. Considerando os 63 com a informação, 49 (77,8\%) haviam apresentado perda de peso nos últimos seis meses. Destes, $31(49,2 \%)$ apresentaram perda significativa de peso e $18(28,5 \%)$ perda de peso grave. Quatorze idosos $(22,2 \%)$ não apresentavam perda de peso nos últimos seis meses. Não houve associação entre percentual de perda de peso e sexo (Tabela 2). 
O estado nutricional de acordo com a MANR foi classificado como desnutrição em 30 (42,9\%) pacientes, como risco de desnutrição em 29 (41,4\%) e como estado nutricional normal em $11(15,7 \%)$. Dessa forma, o percentual de idosos classificados como risco de desnutrição e desnutridos pela MANR ( $\mathrm{n}=59$; $84,3 \%$ ) foi superior ao percentual de desnutrição identificado pelo IMC $(\mathrm{n}=12 ; 17,1 \%)$ e pela $\mathrm{CP}(\mathrm{n}=24$; 34,3\%) (Tabela 2).

Em 63 pacientes foi possível identificar a localização da neoplasia. Os pacientes com câncer de pulmão e pâncreas foram os que mais apresentaram perda de peso grave, enquanto que a maioria das idosas com câncer de mama não apresentou perda de peso. Observou-se que o câncer de pâncreas foi o que resultou em maior porcentagem de pacientes com perda de peso (100\% dos pacientes), seguido pelo de pulmão $(85,6 \%)$, próstata $(72,7 \%)$ e intestino $(69,6 \%)$ (Tabela 3).

\section{DISCUSSÃO}

Observou-se que o período de internação dos pacientes deste estudo foi em geral curto ( 1 a 10 dias), o que pode ser explicado pela rotatividade dos pacientes, que apresentando condições favoráveis ao tratamento internam apenas para realização do mesmo, recebendo alta hospitalar no período planejado, ou que demandam apenas uma intervenção imediata para suporte clínico em casos de emergência. A internação hospitalar é necessária em muitas situações, tendo em vista a complexidade do tratamento, porém segundo Costa, Lima [20] o tratamento em seguimento ambulatorial vem se destacando atualmente, por representar uma estratégia de custo benefício tanto ao paciente como ao setor de saúde.

Pesquisa realizada por Tartari et al. [21] avaliou o perfil nutricional de pacientes oncológicos submetidos à quimioterapia, constatando que o câncer de intestino

Tabela 2. Caracterização nutricional segundo parâmetros antropométricos e subjetivos de idosos oncológicos internados no Hospital São Vicente de Paulo em Passo Fundo, RS, $2014(\mathrm{n}=70)$.

\begin{tabular}{|c|c|c|c|c|c|c|c|}
\hline \multirow{2}{*}{ Parâmetro } & \multirow{2}{*}{ Classificação do estado nutricional } & \multicolumn{2}{|c|}{ Sexo feminino } & \multicolumn{2}{|c|}{ Sexo masculino } & \multirow{2}{*}{$\begin{array}{c}\text { Total } \\
\mathbf{n}\end{array}$} & \multirow{2}{*}{$\mathbf{p}^{*}$} \\
\hline & & $\mathbf{n}$ & $(\%)$ & $\mathbf{n}$ & $(\%)$ & & \\
\hline \multirow{3}{*}{ IMC } & Baixo peso & 9 & $(75)$ & 3 & (25) & 12 & \multirow{3}{*}{$>0,05$} \\
\hline & Eutrofia & 13 & $(33,3)$ & 26 & $(66,7)$ & 39 & \\
\hline & Sobrepeso/obesidade & 8 & $(42,1)$ & 11 & $(57,9)$ & 19 & \\
\hline \multirow{2}{*}{$\mathrm{CP}$} & Desnutrição & 13 & $(54,2)$ & \multirow{2}{*}{1129} & $(45,8)$ & 24 & \multirow{2}{*}{$>0,05$} \\
\hline & Eutrofia & 17 & $(37)$ & & $(63)$ & 46 & \\
\hline \multirow{4}{*}{$\% \mathrm{PP}$} & Perda grave & 12 & $(66,7)$ & 6 & $(33,3)$ & 18 & \multirow{4}{*}{$>0,05$} \\
\hline & Perda significativa & 9 & $(29)$ & 22 & $(71)$ & 31 & \\
\hline & Sem perda de peso & 5 & $(35,7)$ & 9 & $(64,3)$ & 14 & \\
\hline & Não soube informar & 4 & $(57,1)$ & 3 & $(42,8)$ & 7 & \\
\hline \multirow{3}{*}{ MANR } & Desnutrição & 14 & $(46,7)$ & 16 & $(53,3)$ & 30 & \multirow{3}{*}{$>0,05$} \\
\hline & Risco de desnutrição & 13 & $(44,8)$ & 16 & $(55,2)$ & 29 & \\
\hline & Estado nutricional normal & 3 & $(27,3)$ & 8 & $(72,7)$ & 11 & \\
\hline
\end{tabular}

IMC, índice de massa corporal; $\mathrm{CP}$, circinferência da panturrilha; \% PP, porcentagem de perda de peso nos últimos 6 meses (grave $>10 \%$, significativa $1-10 \%$ e sem perda $<1 \%$ ); MANR, Mini Avaliação Nutricional Reduzida.

* Teste do Qui Quadrado.

Tabela 3. Associação entre localização do câncer e perda de peso em idosos oncológicos internados no Hospital São Vicente de Paulo em Passo Fundo, RS, 2014 ( $n=63)$.

\begin{tabular}{|c|c|c|c|c|c|c|}
\hline \multirow{3}{*}{ Local do câncer } & \multicolumn{6}{|c|}{ Perda de peso* } \\
\hline & \multicolumn{2}{|c|}{ Sem perda } & \multicolumn{2}{|c|}{ Perda significativa } & \multicolumn{2}{|c|}{ Perda grave } \\
\hline & $\mathbf{n}$ & $(\%)$ & $\mathbf{n}$ & (\%) & n & $(\%)$ \\
\hline Intestino & 5 & $(31,3)$ & 8 & (50) & 3 & $(18,8)$ \\
\hline Próstata & 3 & $(27,3)$ & 7 & $(63,6)$ & 1 & $(9,1)$ \\
\hline Pulmão & 1 & $(14,3)$ & 2 & $(28,6)$ & 4 & $(57,1)$ \\
\hline Pâncreas & - & - & 3 & (60) & 2 & (40) \\
\hline Mama & 3 & (60) & 1 & (20) & 1 & (20) \\
\hline Outros & 2 & $(10,5)$ & 10 & $(52,6)$ & 7 & $(36,8)$ \\
\hline
\end{tabular}

* Sem perda: porcentagem de perda de peso nos últimos 6 meses $<1 \%$; perda significativa: porcentagem de perda de peso nos últimos 6 meses 1-10\%; perda grave: porcentagem de perda de peso nos últimos 6 meses $>10 \%$. 
foi o mais prevalente, o que também se observou no presente estudo, seguido de próstata, pulmão, pâncreas e mama.

A avaliação nutricional pelo IMC classificou a maioria dos pacientes como eutrófica ou com sobrepeso/ obesidade. Essa classificação pelo IMC pode resultar do fato do surgimento do câncer ser recente, ou porque muitos pacientes com câncer apresentam edema, devido aos procedimentos cirúrgicos ou por efeito de medicamentos. Assim, a realização do IMC isolado para o diagnóstico de desnutrição em indivíduos com câncer tem limitações, tendo em vista que algumas alterações como ascite e edema periférico, podem mascarar a perda de gordura e proteína. É necessária a verificação do estado nutricional de pacientes oncológicos por outros métodos [16,22].

A CP é um indicador de perda de massa muscular. Neste estudo, a maioria dos idosos não apresentou valores que indicassem tal perda, a qual já foi encontrada em outros estudos [21,23], inclusive no Inquérito Luso-Brasileiro de Nutrição Oncológica do Idoso [15]. Apesar do elevado percentual de eutrofia no IMC e na $\mathrm{CP}$, observamos a desnutrição através da MANR, fato este que se justifica devido ao fato da MANR considerar o estado global de saúde.

Em pacientes com câncer a perda de peso é um distúrbio frequente, estando presente em 40 a $80 \%$ dos casos. O grau da perda depende de vários fatores, incluindo o tipo e a localização primária do tumor [24,25]. Na presente amostra, quando classificada pelo percentual de perda de peso, observou-se alta frequência de perda significativa de peso, seguida da perda de peso grave, ao contrário do que foi identificado no estudo de Freitas et al. [26], em que houve predomínio da perda de peso grave $(56,1 \%)$ seguida da perda significativa $(4,9 \%)$.

Verificaram-se percentuais maiores de perda de peso grave no câncer de pulmão e no de pâncreas, e poucos casos de perda de peso relacionados ao câncer de mama. Estes dados assemelham-se aos encontrados por Dewys et al. [27], que analisaram a perda de peso em mais de 3.000 pacientes oncológicos, relacionando com o tipo de tumor, e constataram que a perda de peso grave foi mais frequente no câncer gástrico (33\%) seguido pelo câncer de pâncreas (26\%) e pelo de pulmão (15\%). Naquele estudo também foi encontrada uma baixa prevalência de perda de peso em portadoras de câncer de mama [27].

As neoplasias gastrointestinais interferem mais na nutrição do paciente, pois podem afetar diretamente a alimentação, devido a anomalias na mucosa digestiva e mau funcionamento do aparelho gastrointestinal, causando dificuldades na digestão, má absorção e/ou atraso do esvaziamento gástrico [28]. Já no câncer de mama, nota-se maior prevalência de aumento de peso, o que pode estar relacionado às drogas utilizadas no tratamento desse tumor, às alterações hormonais e aos distúrbios psicológicos [29].

De acordo com a MANR, observou-se alta frequência de desnutrição e risco nutricional na amostra investigada, o que também foi visto pelo Inquérito Luso-Brasileiro de Nutrição Oncológica do Idoso, porém em percentuais inferiores ao encontrado no presente estudo: $39,8 \%$ encontravam-se sob risco de desnutrição e 33,2\% já desnutridos [15].

No presente estudo, utilizou-se a MANR, diferente da maioria dos estudos que utilizam a Mini Avaliação Nutricional (MAN) completa. Foi utilizada a MANR por tratar-se de um estudo com dados secundários, a partir de um formulário já estabelecido pelo INCA. Constatou-se maior prevalência de desnutrição na avaliação pela MANR do que pelo IMC. O estudo de Pereira [30], que utilizou a MAN para avaliar o estado nutricional de idosos institucionalizados, identificou 55,6\% sob risco de desnutrição, 8,3\% com desnutrição e $36,1 \%$ com estado nutricional normal. Naquele estudo, o IMC classificou $10 \%$ dos idosos com baixo peso, valor este inferior ao encontrado no presente estudo. Salienta-se que aquele estudo não incluiu apenas idosos oncológicos [30].

Outros estudos também identificaram que a MAN ou a MANR detectaram com maior precisão o risco de desnutrição do que o valor encontrado pelo IMC. Esses métodos de avaliação nutricional subjetiva são importantes para apontar grupos vulneráveis à desnutrição que necessitam de maior investigação clínica [31,32]. A MANR consiste na aplicação apenas da primeira parte da MAN completa, considerada a triagem do paciente, utilizada inicialmente para avaliar se há risco nutricional. Após realização da mesma, pode-se completar a avaliação apenas, nos pacientes que apresentarem risco nutricional, possibilitando maior praticidade e agilidade na aplicação [33].

Como limitações deste estudo é importante mencionar o número reduzido da amostra e o fato de se tratar de um estudo com dados secundários. Podem ter sido registradas informações de maneira incorreta, ou ter havido perda ou insuficiência de dados para analisar determinadas variáveis. Outra limitação importante do estudo foi a utilização da MANR, uma vez que MAN completa proporcionaria uma avaliação mais detalhada do estado geral de saúde e alimentação dos pacientes idosos oncológicos. Mesmo diante destas limitações, os resultados contribuíram para avaliar o risco nutricional dos pacientes em diversos parâmetros. 
Conclui-se que a maioria dos idosos oncológicos hospitalizados encontrava-se eutrófica pelos indicadores antropométricos IMC e CP, enquanto uma proporção maior desses pacientes foram classificados como desnutridos ou em risco nutricional pela MANR, assim como pela porcentagem de perda de peso. Os pacientes com câncer de pulmão e pâncreas foram os que mais apresentaram perda de peso grave, enquanto que a maioria dos casos de câncer de mama não tinham perda de peso. Os presentes resultados confirmam a importância de usar diferentes parâmetros para avaliar o estado nutricional de idosos oncológicos, de forma a possibilitar uma intervenção nutricional precoce.

\section{NOTA}

Declaração de conflitos de interesse

Os autores declaram não haver conflitos de interesse relevantes ao conteúdo deste estudo.

\section{REFERÊNCIAS}

1. Guedes ACB, Gama CR, Tiussi ACR. Avaliação nutricional subjetiva do idoso: Avaliação Subjetiva Global (ASG) versus Mini Avaliação Nutricional (MAN). Comun Ciênc Saúde. 2008;19(4):377-84.

2. Thun MJ, Delancey JO, Center MM, Jemal A, Ward EM. The global burden of cancer: priorities for prevention. Carcinogenesis. 2010 Jan;31(1):100-10. http://dx.doi.org/10.1093/carcin/bgp263

3. Paltasingh T, Tyagi R. Demographic transition and population ageing: building an inclusive culture. Soc Change. 2012; 42(3):391-409. http://dx.doi.org/10.1177/0049085712454053

4. Molina-Garrido MJ, Guillén-Ponce C. Development of a cancer-specific Comprehensive Geriatric Assessment in a University Hospital in Spain. Crit Rev Oncol Hematol. 2011 Feb;77(2):148-61. http://dx.doi.org/10.1016/j.critrevonc.2010.02.006

5. Ministério da Saúde (BR). DATASUS [Internet]. Informações de Saúde. [citado 2015 Ago 1]. Disponível em: http://www2. datasus.gov.br/DATASUS/index.php

6. Garófolo A. Nutrição Clínica, Funcional e Preventiva Aplicada à Oncologia: Teoria e Prática Profissional. Rio de Janeiro: Rubio; 2012.

7. Lopes LF, Rossi BM, Nakagawa WT. Oncologia cirurgica. In: Kowalslki LP, Anelli LF, Salvajoli JV, Lopes LF. Manual de condutas diagnósticas e terapeuticas em oncologia. $2^{\mathrm{a}}$ ed. São Paulo: Ambito; 2002. p. 81-91.

8. Shils ME, Shike M. Suporte nutricional do paciente com câncer. In: Shils ME, Olson JÁ, Shike M, Ross AC. Tratado de nutrição moderna na saúde e na doença. $9^{\mathrm{a}}$ ed. São Paulo: Manole; 2003. p. 1385-416.

9. Ministério da Saúde (BR). Consenso Nacional de Nutrição Oncológica. Instituto Nacional de Câncer José Alencar Gomes da Silva. Rio de Janeiro: INCA; 2011.

10. Duval PA, Vargas BL, Fripp JC, Arrieira ICO, Lazzeri B, Destri K. Caquexia em pacientes oncológicos internados em um programa de internação domiciliar interdisciplinar. Rev Bras Cancerol. 2010;56(2):207-12.

11. Paillaud E, Liuu E, Laurent M, Le Thuaut A, Vincent H, Raynaud-Simon A, Bastuji-Garin S, Tournigand C, Caillet P, CanouiPoitrine F, on behalf of the ELCAPA Study Groupi. Geriatric syndromes increased the nutritional risk in elderly cancer patients independently from tumoursite and metastatic status. The ELCAPA-05 cohort study. Clin Nutr. 2014 Apr;33(2):330-5. http://dx.doi.org/10.1016/j.clnu.2013.05.014

12. Guigoz Y. The mini nutritional assessment $(\mathrm{MNA} 囚)$ Review of the literature - what does it tell us? J Nutr Health Aging. 2006 Nov-Dec;10(6):466-85; discussion 485-7.

13. Bon AMX, Leung MCA, Galisa MS, Mesquita DM. Atendimento nutricional: uma visão prática. Adultos e idosos. São Paulo: M. Books do Brasil; 2013.

14. Santos ACO, Machado MMO, Leite EM. Envelhecimento e alterações do estado nutricional. Geriatr Gerontol. 2010; 4(3):168-75.

15. Ministério da Saúde (BR). Inquérito luso-brasileiro de nutrição oncológica do idoso: um estudo multicêntrico. Instituto Nacional de Câncer José de Alencar Gomes da Silva. Rio de Janeiro: INCA; 2015.

16. Lipschitz, DA. Screening for nutritional status in the elderly. Prim Care. 1994 Mar;21(1):55-67.

17. Blackburn GL, Bistrian BR, Maini BS, Schlamm HT, Smith MF. Nutritional and metabolic assessment of the hospitalized patient. JPEN J Parenter Enteral Nutr. 1977;1(1):11-22. http://dx.doi.org/10.1177/014860717700100111

18. Chumlea WC, Guo SS, Vellas B, Guigoz Y. Techniques of assessing muscle mass and function (sarcopenia) for epidemiological studies of the elderly. J Gerontol A Biol Sci Med Sci. 1995 Nov;50 Spec No:45-51.

19. Kaiser MJ, Bauer JM, Ramsch C, Uter W, Guigoz Y, Cederholm T, Thomas DR, Anthony P, Charlton KE, Maggio M, Tsai AC, Grathwohl D, Vellas B, Sieber CC; MNA-International Group. Validation of the mini nutritional assessment short-form (mna $\left.{ }^{\circledR}-\mathrm{sf}\right)$ : a practical tool for identification of nutritional status. J Nutr Health Aging. 2009 Nov;13(9):782-8. http://dx.doi. org/10.1007/s12603-009-0214-7 
20. Costa JC, Lima RAG. Crianças/adolescentes em quimioterapia ambulatorial: implicações para a enfermagem. Rev Latinoam Enfermagem. 2002;10(3):321-33. http://dx.doi.org/10.1590/S0104-11692002000300007

21. Tartari RF, Busnello FM, Nunes CHA. Perfil Nutricional de Pacientes em Tratamento Quimioterápico em um Ambulatório Especializado em Quimioterapia. Rev Bras Cancerol. 2010;56(1):43-50.

22. Candela CG, Pena GM, De Cos Blanco AI, Rosado CI, Rabaneda RC. Evaluacion del estado nutricional en el paciente oncologico. SEOM. 2004;4(1):43-56.

23. Martin FG, Nebuloni CC, Najas MS. Correlação entre estado nutricional e força de preensão palmar em idosos. Rev Bras Geriat Gerontol. 2012;15(3):493-504. http://dx.doi.org/10.1590/S1809-98232012000300010

24. Wong PW, Eneiquez A, Barrera R. Nutritional support in critically ill patients with cancer. Crit Care Clin. 2001 Jul;17(3):74367. http://dx.doi.org/10.1016/S0749-0704(05)70206-2

25. Dufau L. Prevalencia de desnutrción en pacientes oncológicos. Diaeta. 2010;28(130):37-44.

26. Freitas BJSA, Mesquita LC, Teive NJV, Souza SR. Antropometria Clássica e Músculo Adutor do Polegar na Determinação do Prognóstico Nutricional em Pacientes Oncológicos. Rev Bras Canc. 2010;56(4):415-22.

27. Dewys WG, Begg C, Lavin PT y cols. Prognostic effect of weight loss prior to chemotherapy in cancer patients. Eastern Cooperative Oncology Group. Am J Med. 1980 Oct;69(4):491-7. http://dx.doi.org/10.1016/S0149-2918(05)80001-3

28. Sociedade Brasileira de Nutrição Parenteral e Enteral. Associação Brasileira de Nutrologia. Terapia nutricional na oncologia. Projeto Diretrizes; 2011.

29. Silva BC, Fernandes RC, Martins KA, Machado MG. Influência da quimioterapia no peso corporal de mulheres com câncer de mama. Comun Ciênc Saúde. 2010;21(3):245-52.

30. Pereira RS. Risco de desnutrição em idosos institucionalizados do município do Rio de Janeiro: um estudo com a mini avaliação nutricional (MAN) [dissertação]. [Rio de Janeiro]: Universidade do Estado do Rio de Janeiro; 2004.

31. Emed TCX, Kronbauer A, Magnoli D. Mini-avaliação Nutricional como indicador de diagnóstico em idosos de asilos. Rev Bras Nutr Clin. 2006;2(3):219-23.

32. Bauer JM, Vogl T, Wicklein S, Trogner J, Muhlberg W, Sieber CC. Comparison of the Mini Nutritional Assessment, Subjective Global Assessment, and Nutritional Risk Screening (NRS 2002) for nutritional screening and assessment in geriatric hospital patients. Z Gerontol Geriatr. 2005 Oct;38(5):322-7. http://dx.doi.org/10.1007/s00391-005-0331-9

33. Ranhoff A, Gjoen AU. Screening for malnutritionin elderly acute medical patients: The usefulness of MNA-SF. J Nutr Health Aging. 2005 Jul-Aug;9(4):221-5. $€$ 